\title{
Fluid dynamics in multiphase distillation processes in packed towers
}

\author{
Andreas Hoffmann*, Ilja Ausner, Jens-Uwe Repke, Günter Wozny \\ TU Berlin, Institute of Process and Plant Technology, Sekr. KWT 9, \\ Strasse des 17. Juni 135, 10623 Berlin, Germany
}

\begin{abstract}
In order to design packed columns for multiphase operation it is necessary to include reliable data on the flow behavior in the model. Since experiments inside packings are very difficult to perform, CFD simulations are considered to be a sensible approach as numerical experiments. To obtain reliable results validation is inevitable. We present the validation of two- and three-phase film flow under transient conditions. This application is not abundant in literature. Numerical results are compared to data from own experiments. A statement is given on the usability of a VOF-like code for such applications.
\end{abstract}

\section{Introduction}

During the last decades packed columns have become popular due to their increase in efficiency and capacity. While these benefits are widely used for distillations with only one liquid phase packed towers are still avoided for processes with more liquid phases. This is mainly related to the fact that the liquid flow behavior changes dramatically with the appearance of a second phase and with that the tower efficiency. Designing packed columns for three-phase distillation requires a reasonable model for the separation efficiency (Repke et al. 2003). While various models exist for two-phase distillation (e.g. Rocha, Bravo, Fair 1993, Brunazzi and Paglianti 1997, Iliuta and Larachi 2001) yet only a few works focus on multiphase operations. One of the main problems is the prediction of the flow behavior inside the column. Most models for the two-phase distillation are based on either film or rivulet flow inside the packed columns. In case of three-phase operation it would be more appropriate to assume film flow for one phase and rivulets or droplets for the other (see fig. 1). Since it is almost impossible to look into the packings simulations with computational fluid dynamics (CFD) will be used to minimize the experimental effort to describe and analyze the influence of various parameters. For these cases no validation of the CFD codes has been done until now. We present the validation of a volume of fluid (VOF) method for two- and three-phase film flows under transient conditions. With validated methods it is possible to develop a model for the effective interfacial area which considers the flow behavior inside packings which is necessary to design packed towers.

* Corresponding author. Tel.: +49 3031423039

E-mail address: Andreas.Hoffmann@TU-Berlin.de 


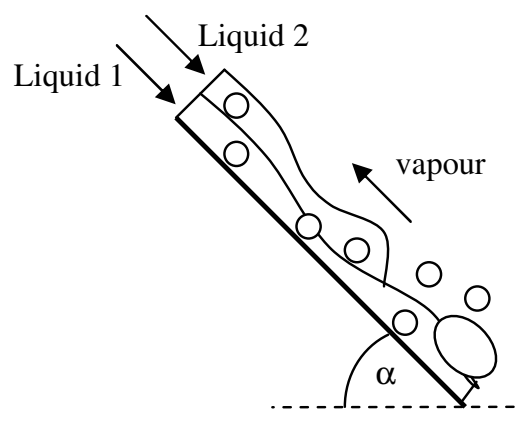

Fig. 1: Possible flow behaviors on a sheet in a packed column

\section{Simulations}

The main goal is to develop a model for the interfacial area between all phases in a multiphase operated packed column. Recent models only consider parameters from binary equations. In three-phase film flow it is not possible to just calculate with two of the phases since the flow behavior and with that the mass transfer strongly changes under the influence of a second liquid phase. Depending on the liquid flow rate it is probable that one of the two liquid phases forms a film and the other phase runs down as droplets or rivulets. Therefore it is necessary that the CFD method is able to predict surface break-up, droplet and rivulet formation properly.

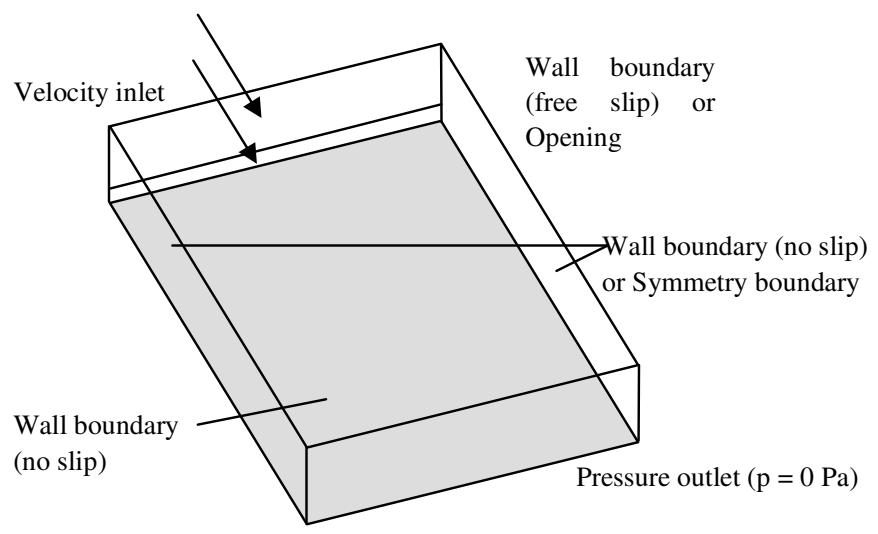

Fig. 2: Model geometry and boundary conditions 
CFD calculations are carried out with the commercial code CFX 5.6, ANSYS Inc., which uses an Euler-Euler algorithm for multiphase calculations with free surfaces. Like the VOF (volume of fluid) method (Hirt and Nichols 1979) it uses avereged phase fractions in the finite volume cells. The surface tension is implemented using the method of Brackbill et al. 1992. The contact angles used are derived from static measurements. It is assumed that the influence of the dynamics on the contact angle is limited to the measuring error range (Stein 1999). Turbulence is not taken into account since the range of the Reynolds-number indicates laminar or pseudolaminar flow $(\operatorname{Re}<$ 350). The model uses a specified velocity profile at the inlet, a wall boundary (no slip, defined contact angles) as the plate, and a pressure outlet (gauge pressure of $p=0$ ) at the outlet. The geometry sides are either implemented as symmetry boundaries or as walls and the upper boundary can be modeled as an opening (inlet and outlet with zero-gaugepressure) or as a wall with free slip (fig. 2). The Reynolds-number Re is defined with the average film-velocity and the film thickness:

$\operatorname{Re}=\frac{\bar{w} \delta}{v}$

This corresponds to the liquid load $\Gamma$ over equation (2):

$\Gamma=\frac{\dot{V}}{b}=\bar{w} \delta=\operatorname{Re} \cdot v$
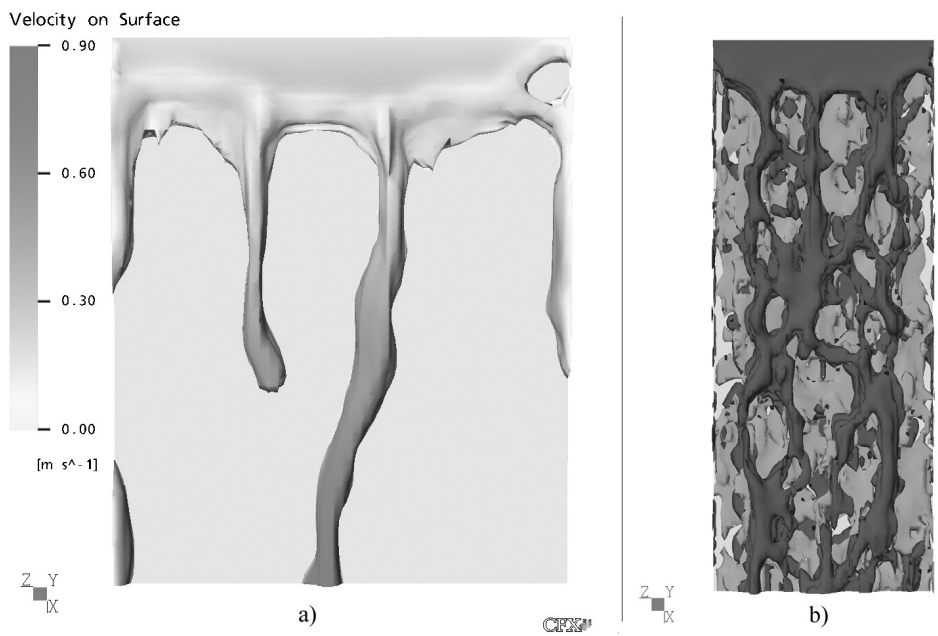

b)

Fig. 3: Simulation: a) momentary result with one liquid phase (water) at a liquid load of $\Gamma=1,0 \mathrm{~cm}^{2} / \mathrm{s}$ or $\operatorname{Re}=112 ; b$ ) momentary result with two liquid phases (toluene: light grey; water: dark grey) at $R e_{W}=250$ and $R e_{T}=220$ 
To get reliable results for model development, it is necessary to validate the CFD code. To our knowledge, this has not been done yet for this application which has various difficulties. Two-phase film flow with free surfaces has already been considered in literature but three-phase film flow with different interfaces is more difficult to simulate. Interaction and surface tension between all phase pairs have to be considered. Two and three dimensional cases are simulated with one and two liquid phases (fig. 3). The main interest lies in the correct prediction of the critical liquid load for the surface break-up and the resulting shadow (or wetted wall) area (example result in fig. 3 a). For the model development it is necessary to calculate the interfacial areas between the fluids. We assume that it is sufficient to define the interface at a specific phase fraction.

\section{Experiments}

The experimental setup is shown in fig. 4. A PTV (particle tracking velocimetry) method is applied to measure film flow velocities and the wetted wall area can be determined by optical means.

The velocities are measured by a PTV method with Isospheres SG-300-B of Omega Minerals Germay GmbH (mean diameter of $110-130 \mu \mathrm{m}$ and density in the range of 0.65 to $0.75 \mathrm{~g} / \mathrm{ccm}$ ). These particles are exposed with a stroboscope with a frequency of $250 \mathrm{~Hz}$. With a CCD-camera with a frequency of 60 images/second this results into a double exposure per image.

To get the shadow area the pictures taken with the CCD-camera are analized with a special image analysis software. Dyes are used to increase the contrast. The applicability of fluorecent dyes to measure the film thickness additionally is examined. The feed tubes shown in fig. 4 can be replaced by an overflowing weir. This setup better resembles the inlet boundary condition of the CFD simulations but is not applicable for two liquid phases.

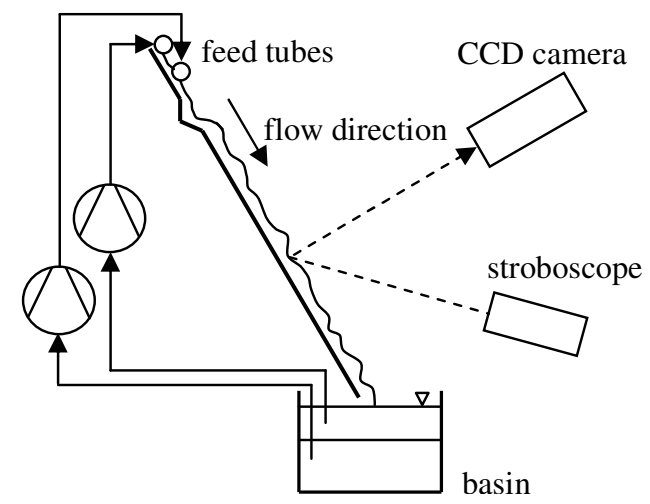

Fig. 4: Experimental setup for the case of two immiscible liquid phases 


\section{Validation}

For validation the simulation results are compared with the data obtained from our experiments and the well known Nusselt solution. Parameters for the validation are e.g. the critical fluid load, film velocities and the wetted wall area. It is not sufficient to consider only one of these parameters.

In fig. 5 the shadow area obtained from simulations and experiments is shown. It can be seen that the shadow area is underestimated or, to put it the other way, that the curve is shifted right about $\operatorname{Re} \sim 50$. The mesh is sufficiently fine, i.e. if we change the mesh size near the plate boundary from a thickness of $0.1 \mathrm{~mm}$ to $0.05 \mathrm{~mm}$ we get a solution close to the previous one. If we consider the contact angle as a validation parameter, since it has been observed that the contact angle is influenced by the surface roughness and the velocity, we see that the solution is only slightly influenced by this value. Nevertheless, the critical liquid load or Reynolds-number at which the film break-up occurs can be predicted better. The surface roughness is not taken into account in the simulations other than by the contact angle.

In cases where the liquid forms a stable film the solution gained by simulation meets the Nusselt solution for laminar film flow. Film thickness and velocities are predicted accurately. It is shown that the simulation results are very sensitive to the model geometry. Two dimensional simulations are not capable of describing some flow effects correctly, like the droplet and rivulet. In case of three-phase flow it was not possible to gain two-dimensional solutions, whereas $3 \mathrm{D}$ simulations show the expected effect of water film break-up (fig. 3 b). More details will be given in the presentation.

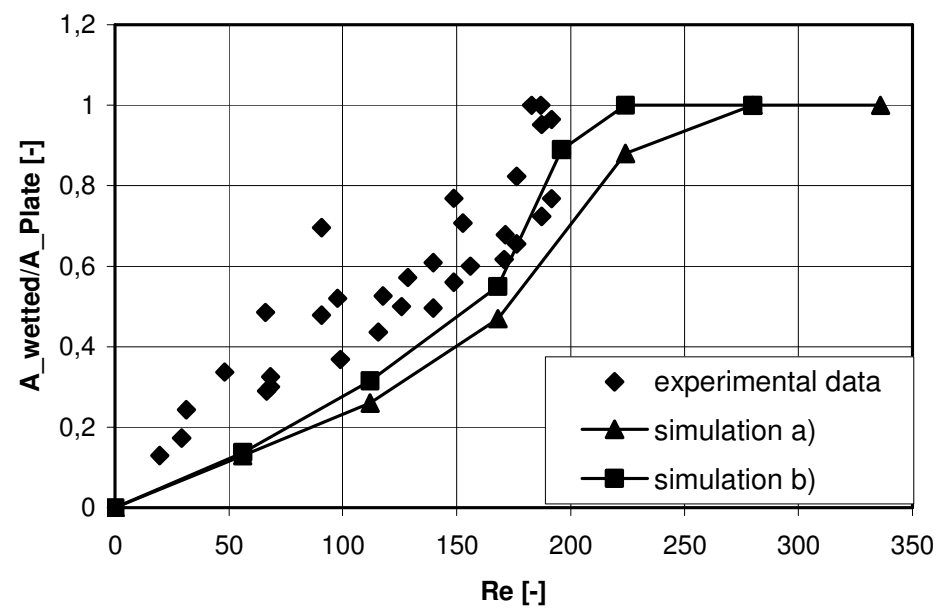

Fig. 5: Comparison of experimental data with numerical results for water flow on an inclined steel plate with an inclination of $60^{\circ}$; the simulation curve a) was obtained with a contact angle of $\theta=80^{\circ}$ the simulation curve $b$ ) with a contact angle of $\theta=70^{\circ}$ 


\section{Conclusion}

To our knowledge, no numerical examination of the flow behaviour in packed columns with more than one liquid phase has been done yet. We present the validation and conclusion on applicability of a CFD code for this application. It is shown that the CFX code is capable of simulating the various flow behaviors. A conclusion is obtained in which range these results are in good agreement with experimental data. Various parameters like the grid size, the contact angle, drag force coefficient and the model parameter interfacial length scale are included in the validation process.

The authors would like to thank the Deutsche Forschungsgemeinschaft (German Research Foundation) for the financial support.

\section{Nomenclature}

$\begin{array}{llll}\text { A_wetted } & \text { wetted area } & \text { A_Plate } & \text { plate area } \\ \mathrm{b} & \text { width of the plate } & \mathrm{p} & \text { pressure } \\ \dot{V} & \text { volume flow rate } & \bar{w} & \text { velocity } \\ \operatorname{Re} & \text { Reynolds-number } & \alpha & \text { inclination angle } \\ \delta & \text { film thickness } & \Gamma & \text { liquid load } \\ \theta & \text { contact angle } & v & \text { kinematic viscosity }\end{array}$

\section{References}

Brackbill, J. U., Kothe, D. B., Zemach, C., 1992, A Continuum Method for Modelling Surface Tension. Journal of Computational Physics 100, pp. 335 - 354

Brunazzi, E., Paglianti, A., 1997, Liquid-Film Mass-Transfer Coefficient in a Column Equipped with Structured Packings. Ind. Eng. Chem. Res. (36), 1997, pp. 3792 3799

Hirt, C. W., Nichols, B. D., 1981, Volume of Fluid (VOF) Method for the Dynamics of Free Boundaries. Journal of Computational Physics 39, pp. 201 - 225

Iliuta, I., Larachi, F., 2001, Mechanistic Model for Structured-Packing-Containg Columns: Irrigated Pressure Drop, Liquid Holdup, and Packing Fractional Wetted Area. Ind. Eng. Chem. Res. (40), 2001, pp. 5140 - 5146

Repke, J.-U., 2002, Experimentelle und theoretische Analyse der Dreiphasenrektifikation in Packungs- und Bodenkolonnen. Fortschritt-Bericht VDI, Reihe 3, Nr. 751 VDI-Verlag Düsseldorf 2002, ISBN 3-18-375103-8

Repke, J.-U., Villain, O., Wozny, G., 2003, A Nonequilibrium Model for Three-Phase Distillation in Packed a Column: Modelling and Experiments. Computer-Aided Chemical Engineering 14, Elsevier, pp. 881-886, ESCAPE-13, Lappeenranta, Finnland, 1.-4.6.2003

Rocha, J. A., Bravo, J. L., Fair, J. R., 1993, Distillation Columns Containing Structured Packings: A Comprehensive Model for Their Performance. 1. Hydraulic Models. Ind. Eng. Chem. Res. 1993 (32), pp. 641 - 651

Stein, W. A., 1999, Benetzung und Zwickelflüssigkeit bei zwei nebeneinander liegenden Zylindern. Forschung im Ingenieurwesen 64, 1999, pp. 329 - 343 